\title{
SMALL DOUBLING IN GROUPS WITH MODERATE TORSION
}

\author{
VSEVOLOD F. LEV
}

\begin{abstract}
We determine the structure of a finite subset $A$ of an abelian group given that $|2 A|<3(1-\varepsilon)|A|, \varepsilon>0$; namely, we show that $A$ is contained either in a "small" one-dimensional coset progression, or in a union of fewer than $\varepsilon^{-1}$ cosets of a finite subgroup.

The bounds $3(1-\varepsilon)|A|$ and $\varepsilon^{-1}$ are best possible in the sense that none of them can be relaxed without tightening another one, and the estimate obtained for the size of the coset progression containing $A$ is sharp.

In the case where the underlying group is infinite cyclic, our result reduces to the wellknown Freiman's $(3 n-3)$-theorem; the former thus can be considered as an extension of the latter onto arbitrary abelian groups, provided that there is "not too much torsion involved".
\end{abstract}

\section{InTRODUCTION AND SUMMARY OF RESUlts}

For subsets $A$ and $B$ of an additively written abelian group, by $A+B$ we denote the set of all group elements representable as $a+b$ with $a \in A$ and $b \in B$. We abbreviate $A+A$ as $2 A$ and define the doubling coefficient of a finite, nonempty set $A$ to be the quotient $|2 A| /|A|$.

It is a basic folklore fact that if $A$ is a finite set of integers, then $|2 A| \geq 2|A|-1$; more generally, if $A$ and $B$ are finite nonempty subsets of a torsion-free abelian group, then $|A+B| \geq|A|+|B|-1$. An extension of this fact onto general abelian groups with torsion is a deep result due to Kneser, discussed in Section 3.

In another direction, Freiman [F62] has established the structure of integer sets $A$ satisfying $|2 A| \leq 3|A|-3$; that is, roughly, sets with the doubling coefficient up to 3 . This result, commonly referred to as Freiman's $(3 n-3)$-theorem, along with its generalizations onto distinct set summands, can be found in any standard additive combinatorics monograph; see, for instance, [N96, Theorem 1.13].

It is a notoriously difficult open problem to merge together the results of Kneser and Freiman establishing the structure of sets with the doubling coefficient less than 3 in abelian groups with torsion. This paper is intended as a step towards the solution of this problem.

Our main result shows that a small-doubling set is either contained in the union of a small number of cosets of a finite subgroup, or otherwise is densely contained in a coset progression. 
Theorem 1. Let $A$ be a finite subset of an abelian group $G$ such that $A$ cannot be covered with fewer than $n$ cosets of a finite subgroup of $G$, for some real $n>0$. If $|2 A|<$ $3\left(1-\frac{1}{n}\right)|A|$, then there exist an arithmetic progression $P \subseteq G$ of size $|P| \geq 3$ and a finite subgroup $K \leq G$ such that $|P+K|=|P||K|, A \subseteq P+K$, and $(|P|-1)|K| \leq|2 A|-|A|$.

Remark 1. The equality $|P+K|=|P||K|$ of Theorem 1 and Theorem 2 below is an easy corollary of the other assertions: if it fails, then there are two elements of $P$ that fall into the same $K$-coset; hence, $P+K$ is a coset of a finite subgroup; therefore $n \leq 1$, which is inconsistent with the assumption $|2 A|<3(1-1 / n)|A|$. For this reason, we ignore the equality in question and never get back to it till the end of the paper.

Remark 2. Letting $\tau=|2 A| /|A|$, the conclusion $(|P|-1)|K| \leq|2 A|-|A|$ can be rewritten as $|P+K| \leq(\tau-1)|A|+|K|$; the meaning of this estimate is that $A$ is dense in $P+K$.

We derive Theorem 1 from the following, essentially equivalent, result.

Theorem 2. Suppose that the abelian group $G$ has the direct sum decomposition $G=$ $\mathbb{Z} \oplus H$ with $H<G$ finite. Let $A \subseteq G$ be a finite set, and let $n$ be number of elements of the image of $A$ under the projection $G \rightarrow \mathbb{Z}$ along $H$. If $|2 A|<3\left(1-\frac{1}{n}\right)|A|$, then there exist an arithmetic progression $P \subseteq G$ of size $3 \leq|P| \leq(\tau-1) n+1$ and a subgroup $K \leq H$ such that $|P+K|=|P||K|, A \subseteq P+K$, and $(|P|-1)|K| \leq|2 A|-|A|$.

The equality $G=\mathbb{Z} \oplus H$ means that $G$ is the direct sum of its infinite cyclic subgroup and the subgroup $H$. To simplify the notation, the former is identified with the group of integers.

The following example shows that Theorem 2 is sharp in the sense that the assumption $|2 A|<3\left(1-\frac{1}{n}\right)|A|$ cannot be relaxed, and the conclusion $(|P|-1)|K| \leq|2 A|-|A|$ cannot be strengthened.

Example 1. Let $P:=[0, l]$ and $A:=([0, n-2] \cup\{l\})+K$, where $K \leq H$, and $l \geq n-1 \geq 2$ are integers; thus, $|A|=n|K|$. If $l>2 n-3$, then $|2 A|=(3 n-3)|K|=3\left(1-n^{-1}\right)|A|$, and $A$ fails to have the structure described in Theorem 3 as $|2 A|-|A|=(2 n-3)|K|<$ $(|P|-1)|K|$. Thus, to conclude that a set $A \subseteq \mathbb{Z} \oplus H$ with $|2 A|<3(1-\varepsilon)|A|$ is densely contained in a coset progression, one needs to assume that $A$ cannot be covered with fewer than $\varepsilon^{-1}$ cosets of a finite subgroup (or make some other extra assumption).

On the other hand, if $l \leq 2 n-3$, then $|2 A|=(l+n)|K|$; therefore, $|2 A|-|A|=$ $(|P|-1)|K|$.

Remark 3. The inequality $|P| \geq 3$ of Theorem 2 follows in fact automatically from the other assertions of the theorem. We cannot have $|P|=1$ because this would lead to $n=1$, and consequently to $|2 A|<0$. We also cannot have $|P|=2$ because this would result in $n=2$ and $|2 A|<\frac{3}{2}|A|$. The latter, in its turn, is known to imply (see, for instance, Lemma 1 below) that $A$ is contained in a coset of a finite subgroup of $G$; hence, 
in an $H$-coset. This, however, contradicts the equality $n=2$. The same applies to Theorem 1.

Similarly, the upper-bound estimate $|P| \leq(\tau-1) n+1$ in Theorem 2 follows from

$$
(\tau-1)|A|=|2 A|-|A| \geq(|P|-1)|K| \geq(|P|-1) \frac{|A|}{n},
$$

and we thus can safely forget this estimate for the rest of the paper.

Remark 4. In the particular case where $H$ is trivial, and $A$ is a subset of the infinite cyclic group, Theorem 2 is equivalent to Freiman's classical $(3 n-3)$-theorem, see [F62] or [N96, Theorem 1.13]. Theorem 2 thus can be considered as an extension of the $(3 n-3)$-theorem onto the groups with torsion.

Remark 5. As a corollary of Theorem 2, for any finite set $A \subseteq \mathbb{Z} \oplus H$, denoting by $n$ the size of the projection of $A$ onto $\mathbb{Z}$ along $H$, we have $|2 A| \geq\left(2-\frac{1}{n}\right)|A|$. This follows by letting $\tau:=|2 A| /|A|$ and observing that

$$
(\tau-1)|A|=|2 A|-|A| \geq(|P|-1)|K| \geq(n-1)|K| \geq\left(1-\frac{1}{n}\right)|A| .
$$

We remark that, while the resulting estimate $|2 A| \geq\left(2-\frac{1}{n}\right)|A|$ may not be completely trivial, it is not particularly deep either, and can be proved independently of the theorem, with a simple combinatorial reasoning in the spirit of the proof of Lemma 6 (Section 4).

It should be possible to use our method to treat sumsets of the form $A+B$ with the distinct set summands, and in particular to prove analogues of Theorems 1 and 2 for the difference sets $A-A$.

Theorem 2 can be compared against the following result of Balasubramanian and Pandey, which is an elaboration on an earlier result of Deshouillers and Freiman [DF86, Theorem 2].

Theorem 3 (Balasubramanian-Pandey [BP18, Theorem 5]). Let $d \geq 2$ be an integer and suppose that $A \subseteq \mathbb{Z} \oplus(\mathbb{Z} / d \mathbb{Z})$ is a finite set with $|2 A|<2.5|A|$. For $z \in \mathbb{Z}$, let $A_{z}:=A \cap(z+\mathbb{Z} / d \mathbb{Z})$, and let $B:=\left\{z \in \mathbb{Z}: A_{z} \neq \varnothing\right\}$. If $|B| \geq 6$ and $\operatorname{gcd}(B-B)=1$, then there exists a subgroup $K \leq \mathbb{Z} / d \mathbb{Z}$ and elements $x, y \in \mathbb{Z} / d \mathbb{Z}$ such that, letting $l:=\max B-\min B$, we have

i) $A \subseteq\{(b, b x+y): b \in B\}+K$;

ii) there exists $b \in B$ with $\left|A_{b}\right| \geq \frac{2}{3}|K|$;

iii) $l|K| \leq|2 A|-|A|$.

Balasubramanian and Pandey also include into the statement the estimate $l<\frac{3}{2}|B|$, but in fact this estimate follows easily from i) and iii):

$$
l|K|<2.5|A|-|A|=\frac{3}{2}|A| \leq \frac{3}{2}|B||K| .
$$


In the same vein, i) and iii) imply an estimate which is only slightly weaker than ii): namely, by iii) we have $l|K| \leq(\tau-1)|A|$; therefore, by averaging, there exists an element $b \in B$ with

$$
\left|A_{b}\right| \geq \frac{|A|}{|B|} \geq \frac{(\tau-1)|A|}{(\tau-1)(l+1)} \geq \frac{l}{l+1} \frac{|K|}{\tau-1} \geq \frac{2}{3}\left(1-\frac{1}{l+1}\right)|K| .
$$

To match the Balasubramanian-Pandey estimate $\max _{b \in B}\left|A_{b}\right| \geq \frac{2}{3}|K|$, we prove in Section 2 the following theorem showing (subject to Theorem 2) that if $n$ is sufficiently large, then there exists a $K$-coset containing at least $\frac{|K|}{\tau-1}$ elements of $A$.

Theorem 4. Suppose that $G, H, A, n, P$, and $K$ are as in Theorem 2, and let $\tau:=$ $|2 A| /|A|$. If $n \geq \frac{4 \tau-6}{(\tau-2)(3-\tau)}$, then there exists a $K$-coset containing at least $\frac{|K|}{\tau-1}$ elements of $A$.

Compared to Theorem 3, our Theorem 2 applies to the groups $\mathbb{Z} \oplus H$ with $H$ not necessarily cyclic and, most importantly, allows the doubling coefficient to be as large as $3-o(1)$ (instead of 2.5), which is best possible, as shown above.

The layout of the remaining part of the paper is as follows. In Section 2 we deduce Theorems 1 and 4 from Theorem 2, allowing us to concentrate on the proof of the latter theorem for the rest of the paper. In Section 3 we collect some general results needed for the proof; in particular, we introduce and briefly discuss Kneser's theorem. In Section 4 we prove some basic estimates related to the particular settings of Theorem 2 (in contrast with Section 3 where the results are of general nature). Section 5 contains a result which, essentially, establishes the special case of Theorem 2 where the set $A$ can be partitioned into two "additively independent" subsets. Finally, we prove Theorem 2 in Section 6.

\section{Deduction of Theorems 1 And 4 From Theorem 2}

Proof of Theorem 1. Let $A$ be a finite subset of an abelian group $G$ such that $A$ cannot be covered with fewer than $n$ cosets of a finite subgroup of $G$, while

$$
|2 A|<3\left(1-\frac{1}{n}\right)|A|
$$

with a real $n>0$. We want to prove, assuming Theorem 2 , that there exist an arithmetic progression $P \subseteq G$ and a subgroup $K \leq G$ such that $A \subseteq P+K$ and $(|P|-1)|K| \leq$ $|2 A|-|A|$. As explained in Section 1 (Remark 3), the progression will satisfy $|P| \geq 3$ and $|P+K|=|P||K|$.

Without loss of generality, we assume that $G$ is generated by $A$. By the fundamental theorem of finitely generated abelian groups, there is then an integer $r \geq 0$ and a finite subgroup $H \leq G$ such that $G \cong \mathbb{Z}^{r} \oplus H$. Indeed, we have $r \geq 1$ as otherwise $G$ would be finite; hence, $A$ would be contained in just one single finite coset (the group $G$ itself), forcing $n \leq 1$ and thus contradicting the small-doubling assumption (1). 
Let $G^{\prime}:=\mathbb{Z} \oplus H$. To avoid confusion, throughout the proof we use the direct product notation for the elements of the groups $G$ and $G^{\prime}$.

Fix an integer $M>0$ divisible by all positive integers up to $|2 A|$, and consider the mapping $\psi: G \rightarrow G^{\prime}$ defined by

$$
\psi\left(x_{1}, \ldots, x_{r}, h\right):=\left(x_{1}+M x_{2}+\cdots+M^{r-1} x_{r}, h\right) ; \quad x_{1}, \ldots, x_{r} \in \mathbb{Z}, h \in H .
$$

If $M$ is large enough (as we assume below), then different elements of $A$ have different images under $\psi$, and similarly for $2 A$; consequently, writing $A^{\prime}:=\psi(A)$, we have $\left|A^{\prime}\right|=$ $|A|$ and $\left|2 A^{\prime}\right|=|2 A|$, whence

$$
\left|2 A^{\prime}\right|<3\left(1-\frac{1}{n}\right)\left|A^{\prime}\right|
$$

(we implicitly use here the equality $2 \psi(A)=\psi(2 A)$ ).

Denote by $m$ the number of elements of the projection of $A$ onto the first (torsion-free) component of $G$. If $M$ is sufficiently large, then this is also the number of elements of the projection of $A^{\prime}$ onto the first component of $G^{\prime}$. Since $A$ is not contained in a union of fewer than $n$ cosets, we have $m \geq n$, resulting in

$$
\left|2 A^{\prime}\right|<3\left(1-\frac{1}{m}\right)\left|A^{\prime}\right|
$$

Applying Theorem 2, we conclude that there exist a finite arithmetic progression $P^{\prime} \subseteq G^{\prime}$ and a subgroup $K \leq H$ such that $A^{\prime} \subseteq P^{\prime}+K$ and

$$
\left(\left|P^{\prime}\right|-1\right)|K| \leq\left|2 A^{\prime}\right|-\left|A^{\prime}\right|=|2 A|-|A| .
$$

We assume that $P^{\prime}$ is the shortest progression possible with $A^{\prime} \subseteq P^{\prime}+K$.

Write $N:=\left|P^{\prime}\right|-1$, and let $c \in G^{\prime}$ and $(d, h) \in G^{\prime}$ denote the initial term and the difference of the progression $P^{\prime}$, respectively; thus,

$$
P^{\prime}=c+\{j(d, h): j \in[0, N]\} ; \quad d \in \mathbb{Z}, h \in H .
$$

Notice that $d \neq 0$, as otherwise we would have $A^{\prime} \subseteq P^{\prime}+K \subseteq c+H$, as a result of which $A^{\prime}$, and therefore also $A$, would be contained in a single $H$-coset.

Since $P^{\prime}$ is the shortest possible progression with $A^{\prime} \subseteq P^{\prime}+K$, there are elements $\left(a_{1}, \ldots, a_{r}, f\right),\left(b_{1}, \ldots, b_{r}, g\right) \in A$ such that $\psi\left(a_{1}, \ldots, a_{r}, f\right)=c$ and $\psi\left(b_{1}, \ldots, b_{r}, g\right)=$ $c+N(d, h) ;$ consequently,

$$
\left(b_{1}-a_{1}\right)+M\left(b_{2}-a_{2}\right)+\cdots+M^{r-1}\left(b_{r}-a_{r}\right)=N d .
$$

Since $N=\left|P^{\prime}\right|-1 \leq|2 A|-|A|<|2 A|$, and recalling that $M$ was chosen to be divisible by all integers up to $|2 A|$, we have $N \mid M$, and therefore $b_{1}-a_{1}$ is a multiple of $N$. Thus

$$
d=\left(b_{1}-a_{1}\right) N^{-1}+M N^{-1}\left(b_{2}-a_{2}\right)+\cdots+M^{r-1} N^{-1}\left(b_{r}-a_{r}\right),
$$

where all summands in the right-hand side are integers. 
We know that for any element $\left(\alpha_{1}, \ldots, \alpha_{r}, \eta\right) \in A$, there exist $j \in[0, N]$ and $k \in K$ such that

$$
\begin{aligned}
\left(\alpha_{1}+\cdots+M^{r-1} \alpha_{r}, \eta\right) & =c+j(d, h)+(0, k) \\
& =\left(a_{1}+\cdots+M^{r-1} a_{r}, f\right)+j(d, h)+(0, k) .
\end{aligned}
$$

Recalling (3), we obtain

$$
\left(\alpha_{1}-a_{1}\right)+\cdots+M^{r-1}\left(\alpha_{r}-a_{r}\right)=j d=j\left(b_{1}-a_{1}\right) N^{-1}+\cdots+j M^{r-1} N^{-1}\left(b_{r}-a_{r}\right) ;
$$

that is,

$$
\left(\alpha_{1}-a_{1}\right) N+\cdots+M^{r-1}\left(\alpha_{r}-a_{r}\right) N=j\left(b_{1}-a_{1}\right)+\cdots+j M^{r-1}\left(b_{r}-a_{r}\right)
$$

with $j \in[0, N]$ depending on $\alpha_{1}, \ldots, \alpha_{r}$. (Notice that $N$ depends on $M$, but is bounded: $N \leq N|K| \leq|2 A|-|A|$ by (2).) Choosing $M$ sufficiently large, from (4) we get

$$
\left(\alpha_{i}-a_{i}\right) N=j\left(b_{i}-a_{i}\right), 1 \leq i \leq r,
$$

showing that $\left(b_{i}-a_{i}\right) j$ is divisible by $N$. Using again the fact that $P^{\prime}$ is the shortest possible progression with $A^{\prime} \subseteq P^{\prime}+K$, we conclude that the possible values of $j$ that can emerge from different elements $\left(\alpha_{1}, \ldots, \alpha_{r}, \eta\right) \in A$ are coprime. Hence, there is a linear combination of these values, with integer coefficients, which is equal to 1 . Consequently, from (5), all numbers $\left(b_{i}-a_{i}\right) N^{-1}, 1 \leq i \leq r$, are integers, and then, by (5) again,

$$
\left(\alpha_{1}, \ldots, \alpha_{r}, \eta\right)=\left(a_{1}, \ldots, a_{r}, f\right)+j\left(\left(b_{1}-a_{1}\right) N^{-1}, \ldots,\left(b_{r}-a_{r}\right) N^{-1}, h\right)+(0, \ldots, 0, k) .
$$

This shows that $A \subseteq P+K$, where $P \subseteq G$ is the $(N+1)$-term arithmetic progression with the initial term $\left(a_{1}, \ldots, a_{r}, f\right)$ and the difference $\left(\left(b_{1}-a_{1}\right) N^{-1}, \ldots,\left(b_{r}-a_{r}\right) N^{-1}, h\right)$. Finally, by (2),

$$
|2 A|-|A|=\left|2 A^{\prime}\right|-\left|A^{\prime}\right| \geq\left(\left|P^{\prime}\right|-1\right)|K|=(|P|-1)|K| .
$$

Proof of Theorem 4. Let $B$ denote the projection of $A$ onto $\mathbb{Z}$ along $H$; thus, $|P| \geq|B|=$ $n$, with equality if and only if $B$ is an arithmetic progression. If this is not the case, then $|P| \geq n+1$ and, by averaging, there is a $K$-coset containing at least

$$
\frac{|A|}{n} \geq \frac{|A|}{|P|-1} \geq \frac{|K|}{\tau-1}
$$

elements of $A$ (the last inequality following directly from the estimate $|2 A|-|A| \geq$ $(|P|-1)|K|$ of Theorem 2). Suppose thus that $B$ is an arithmetic progression and, consequently, $|P|=n$ and $|2 A|-|A| \geq(n-1)|K|$, whence

$$
|A| \geq \frac{n-1}{\tau-1}|K|
$$


Let

$$
\begin{gathered}
M:=\max \{|A \cap(g+K)|: g \in P\}, \mu:=|M| /|K| \\
P_{0}:=\left\{g \in P:|A \cap(g+K)| \leq \frac{1}{2}|K|\right\}, P_{1}:=P \backslash P_{0} \text {, and } m:=\left|P_{0}\right| .
\end{gathered}
$$

Notice that $M>\frac{1}{2}|K|$ as otherwise we would have

$$
\frac{1}{2}|K| \geq M \geq \frac{|A|}{n} \geq\left(1-\frac{1}{n}\right) \frac{|K|}{\tau-1},
$$

which is easily seen to contradict $\tau<3\left(1-\frac{1}{n}\right)$. Therefore $P_{1}$ is nonempty, and $m<n$.

We want to show that $\mu>\frac{1}{\tau-1}$. Suppose for a contradiction that this is wrong. Since $P+K$ is a union of $n$ pairwise disjoint $K$-cosets, of which $m$ contain at most $\frac{1}{2}|K|$ elements of $A$, and the remaining $n-m$ contain at most $M$ elements each, we have

$$
\frac{n-1}{\tau-1}|K| \leq|A| \leq m \cdot \frac{1}{2}|K|+(n-m) \cdot M,
$$

leading to

$$
\frac{n-1}{\tau-1} \leq \frac{1}{2} m+(n-m) \mu<\frac{1}{2} m+\frac{n-m}{\tau-1},
$$

where the last inequality follows from the assumption $\mu<1 /(\tau-1)$. This simplifies to the estimate

$$
m<\frac{2}{3-\tau}
$$

which we will need shortly.

The set $2 P_{1}+K$ is a union of $\left|2 P_{1}\right| \geq 2\left|P_{1}\right|-1=2(n-m)-1$ distinct $K$-cosets contained in $2 A$ by the pigeonhole principle. The set $P+P_{1}+K$ is a union of $\left|P+P_{1}\right| \geq$ $|P|+\left|P_{1}\right|-1=2 n-m-1$ distinct $K$-cosets, each of them containing at least $\frac{1}{2}|K|$ elements of $2 A$. We thus can find $2 n-2 m-1$ cosets represented by the elements of $2 P_{1}$, and then $m$ more cosets represented by the elements of $P+P_{1}$. Altogether, we get $2 n-m-1$ cosets containing at least

$$
(2 n-2 m-1)|K|+\frac{1}{2}|K| m=\left(2 n-\frac{3}{2} m-1\right)|K|
$$

elements of $2 A$. It follows that

$$
2 n-\frac{3}{2} m-1 \leq \frac{|2 A|}{|K|}=\tau \frac{|A|}{|K|} \leq\left(\frac{1}{2} m+(n-m) \mu\right) \tau<\left(\frac{1}{2} m+\frac{n-m}{\tau-1}\right) \tau,
$$

cf. (6). Rearranging the terms gives

$$
\left(1-\frac{1}{\tau-1}\right) n<\left(\frac{3}{2}+\frac{\tau}{2}-\frac{\tau}{\tau-1}\right) m+1
$$

that is, using (7),

$$
\frac{\tau-2}{\tau-1} n<\frac{\tau^{2}-3}{2(\tau-1)} m+1<\frac{\tau^{2}-3}{(\tau-1)(3-\tau)}+1
$$


leading to

$$
n<\frac{\tau^{2}-3}{(\tau-2)(3-\tau)}+\frac{\tau-1}{\tau-2}=\frac{4 \tau-6}{(\tau-2)(3-\tau)},
$$

and the assertion follows.

The rest of the paper is devoted to the proof of Theorem 2 .

\section{General Results}

In this section we collect some general results valid in any abelian group, regardless of the particular settings of Theorem 2.

For a subset $S$ of an abelian group, let $\pi(S)$ denote the period (stabilizer) of $S$; that is, $\pi(S)$ is the subgroup consisting of all those group elements $g$ with $S+g=S$. The set $S$ is called aperiodic or periodic according to whether $\pi(S)$ is or is not the zero subgroup.

We start with a basic theorem due to Kneser which is heavily used in our argument.

Theorem 5 (Kneser, [Kn53, Kn55]; see also [N96, Theorem 4.1]). If $B$ and $C$ are finite, non-empty subsets of an abelian group with

$$
|B+C| \leq|B|+|C|-1,
$$

then letting $L:=\pi(B+C)$ we have

$$
|B+C|=|B+L|+|C+L|-|L| .
$$

We will be referring Theorem 5 as Kneser's theorem.

Since, in the above notation, we have $|B+L| \geq|B|$ and $|C+L| \geq|C|$, Kneser's theorem shows that $|B+C| \geq|B|+|C|-|L|$, leading to

Corollary 1. If $B$ and $C$ are finite, non-empty subsets of an abelian group, such that $|B+C|<|B|+|C|-1$, then $B+C$ is periodic.

The following lemma is well known, but tracing it down to the origin is hardly possible.

Lemma 1. Let $B$ be a finite subset of an abelian group. If $|2 B|<\frac{3}{2}|B|$, then there is a subgroup $L$ such that $B-B=L$, and $2 B$ is an $L$-coset (as a result of which $B$ is contained in a unique $L$-coset).

We give a somewhat nonstandard, self-contained proof of the lemma.

Proof of Lemma 1. For a group element $g$, denote by $r(g)$ the number of representations of $g$ as a difference of two elements of $B$. If $g \in B-B$, then choosing arbitrarily $b, c \in B$ with $g=b-c$ we get

$$
r(g)=|(b+B) \cap(c+B)| \geq 2|B|-|2 B|>\frac{1}{2}|B| .
$$

By the pigeonhole principle, for any $g_{1}, g_{2} \in B-B$ there are representations $g_{1}=$ $b_{1}-c_{1}, g_{2}=b_{2}-c_{2}$ with $c_{1}=c_{2}$; consequently, $g_{1}-g_{2}=b_{1}-b_{2} \in B-B$, showing that $L:=B-B$ is a subgroup. Clearly, $B$ is contained in a unique $L$-coset. 
As we have shown, for every element $g \in L=B-B$ we have $r(g)>\frac{1}{2}|B|$. As a result,

$$
|B|(|B|-1)=\sum_{g \in L \backslash\{0\}} r(g)>\frac{1}{2}|B| \cdot(|L|-1),
$$

implying $|B|>\frac{1}{2}|L|$. Recalling that $B$ is contained in a unique $L$-coset, and using the pigeonhole principle again, we conclude that $2 B$ is an $L$-coset.

Lemma 2. Suppose that $B$ is a subset of an abelian group with $0 \in B$. If $N \geq 3$ is an integer such that $|B|=N+1$ and $|2 B|=2 N+1$ (thus $|2 B \backslash B|=N$ ), then one of the following holds:

i) there exist $b_{1}, \ldots, c_{N} \in B$ such that $2 B \backslash B=\left\{b_{1}+c_{1}, \ldots, b_{N}+c_{N}\right\}$, and every element of $B$ appears among $b_{1}, \ldots, c_{N}$ at most $N$ times;

ii) there is a subgroup $L$ with $|L|=N$ and a group element $g$ with $2 g \notin L$ such that $B=L \cup\{g\}$. (In this case there exist $b_{1}, \ldots, c_{N} \in B$ such that $2 B \backslash B=$ $\left\{b_{1}+c_{1}, \ldots, b_{N}+c_{N}\right\}$, and every element of $B$ appears among $b_{1}, \ldots, c_{N}$ exactly once, except that 0 does not appear at all, and $g$ appears $N+1$ times.)

iii) $N=2$ and there is a subgroup $L$ with $|L|=2$ and a group element $g$ with $2 g \notin L$ such that $B=(g+L) \cup\{0\}$.

Proof. Leaving the case $N=2$ to the reader (hint: write $B=\{0, b, g\}$ and consider two cases: $b+g=0$ and $b+g \neq 0$ ), we confine ourself to the general case where $N \geq 3$.

Choose $b_{1}, \ldots, c_{N} \in B$ arbitrarily to have $2 B \backslash B=\left\{b_{1}+c_{1}, \ldots, b_{N}+c_{N}\right\}$. Since all sums $b_{i}+c_{i}$ are distinct, for any $g \in B$ there is at most one index $i \in[1, N]$ with $b_{i}=c_{i}=g$. Consequently, if there is an element $g \in B$ which appears at least $N+1$ times among $b_{1}, \ldots, c_{N}$ (as we now assume), then in fact it appears $N+1$ times exactly: namely, $b_{i}=c_{i}=g$ for some $i \in[1, N]$ and, besides, for any $j \neq i$, exactly one of $b_{i}$ and $c_{j}$ is equal to $g$. Redenoting, we assume that $b_{1}=c_{1}=\cdots=c_{N}=g$.

Notice that $2 g=b_{1}+c_{1} \in 2 B \backslash B$ along with $0 \in B$ show that $g \neq 0$. Write $B_{0}:=B \backslash\{0\}$ and $B_{g}:=B \backslash\{g\}$. Since the sums $b_{i}+c_{i}=b_{i}+g$ are pairwise distinct, so are the elements $b_{1}, \ldots, b_{N} \in B$. Moreover, $b_{1}, \ldots, b_{N}$ are nonzero in view of $b_{i}+g=b_{i}+c_{i} \notin B$ and $g \in B$, and since $\left|B_{0}\right|=N$, it follows that $\left\{b_{1}, \ldots, b_{N}\right\}=B_{0}$; consequently, $2 B \backslash B=g+B_{0}$.

If there exist some $b, c \in B_{g}$ with $b+c \notin B$, then choosing $i \in[1, N]$ with $b_{i}+g=b+c$ and replacing $b_{i}$ with $b$ and $c_{i}$ with $c$ in the $2 N$-tuple $\left(b_{1}, \ldots, c_{N}\right)$, we get another $2 N$ tuple $\left(b_{1}^{\prime}, \ldots, c_{N}^{\prime}\right)$ such that the sums $b_{i}^{\prime}+c_{i}^{\prime}$ list all elements of $2 B \backslash B$. If $i \in[2, N]$, then $g$ appears exactly $N$ times among $b_{1}^{\prime}, \ldots, c_{N}^{\prime}$, so that no other element of $B$ can appear $N+1$ or more times. Similarly, if $i=1$, then in view of $c_{2}^{\prime}=\cdots=c_{N}^{\prime}=g$, and since all sums $b_{2}^{\prime}+c_{2}^{\prime}, \ldots, b_{N}^{\prime}+c_{N}^{\prime}$ are pairwise distinct, every element $b \in B_{g}$ appears at most $3<N+1$ times among $b_{1}^{\prime}, \ldots, c_{N}^{\prime}$. Thus, the assertion holds true in this case.

Suppose therefore that $b, c \in B_{g}$ with $b+c \notin B$ do not exist; that is, $2 B_{g} \subseteq B$. This gives $\left|2 B_{g}\right| \leq\left|B_{g}\right|+1$; hence, by Lemma 1 and in view of $0 \in B_{g}$, the set $L:=B_{g}-B_{g}=$ $2 B_{g}$ is a subgroup. Furthermore, since $B_{g} \subseteq 2 B_{g}=L$ and $\left|B_{g}\right| \geq\left|2 B_{g}\right|-1=|L|-1$, we 
have either $B_{g}=L$, or $B_{g}=L \backslash\{l\}$ with some $l \in L, l \neq 0$. The latter case is in fact impossible as we would have $l \in 2 B_{g} \backslash B$ in this case, contradicting the present assumption $2 B_{g} \subseteq B$. In the former case we have $B=L \cup\{g\}$ and $2 B=L \cup(g+L) \cup\{2 g\}$, with $2 g \notin L$ in view of $|2 B|=2 N+1=2|B|-1=2|L|+1$.

Lemma 3. Suppose that $L$ is a subgroup, and that $B$ and $C$ are subsets of an abelian group. Let $\varphi_{L}$ denote the canonical homomorphism onto the quotient group.

i) We have $\varphi_{L}(B \cup C)=\varphi_{L}(B) \cup \varphi_{L}(C)$.

ii) If at least one of $B+L=B$ and $C+L=C$ holds, then $\varphi_{L}(B \cap C)=\varphi_{L}(B) \cap$ $\varphi_{L}(C)$, and hence $\varphi_{L}(B \backslash C)=\varphi_{L}(B) \backslash \varphi_{L}(C)$.

Proof. The first assertion is trivial and is stated for completeness only.

For the second assertion, we assume, for definiteness, that $B+L=B$, and show that $\varphi_{L}(B) \cap \varphi_{L}(C) \subseteq \varphi_{L}(B \cap C)$; the opposite inclusion is trivial. Fix an element $t \in \varphi_{L}(B) \cap \varphi_{L}(C)$. Since $t \in \varphi_{L}(C)$, there exists $c \in C$ with $t=\varphi_{L}(c)$. Now $\varphi_{L}(c)=t \in$ $\varphi_{L}(B)$ gives $c \in B+L=B$, showing that $c \in B \cap C$ and therefore $t=\varphi_{L}(c) \in \varphi_{L}(B \cap C)$. The assertion follows.

Corollary 2. Suppose that $L$ is a subgroup, and that $B$ and $C$ are subsets of an abelian group. If $B+L=B$, then $\varphi_{L}(B \cap C)=\varphi_{L}(B)$ is equivalent to any of $\varphi_{L}(B) \subseteq \varphi_{L}(C)$ and $B+L \subseteq C$.

Proof. Applying the lemma, we get $\varphi_{L}(B \cap C)=\varphi_{L}(B) \cap \varphi_{L}(C)$. Thus, $\varphi_{L}(B \cap C)=$ $\varphi_{L}(B)$ is equivalent to $\varphi_{L}(B) \subseteq \varphi_{L}(C)$, which is immediately seen to be equivalent to $B \subseteq C+L$.

Lemma 4. If $G$ is an abelian group having the direct sum decomposition $G=\mathbb{Z} \oplus H$ with $H$ finite, then every subgroup $G^{\prime}<G$ is of the form $G^{\prime}=\langle g\rangle+K$ with some $g \in G$ and $K \leq H$; indeed, one can take $K:=G^{\prime} \cap H$.

Proof. The assertion is immediate if $G^{\prime} \leq H$; assume therefore that $G^{\prime} \not \leq H$. In this case the projection of $G^{\prime}$ onto $\mathbb{Z}$ along $H$ is a non-zero subgroup of $\mathbb{Z}$; let $z^{\prime}$ be its generator. For $k \in \mathbb{Z}$, the "slice" $G^{\prime}(k):=G^{\prime} \cap(k+H)$ is non-empty if and only if $z^{\prime} \mid k$. Furthermore, for any $k_{1}, k_{2}$ divisible by $z^{\prime}$, and any fixed $d \in G^{\prime}\left(k_{2}-k_{1}\right)$, we have $G^{\prime}\left(k_{1}\right)+d \subseteq G^{\prime}\left(k_{2}\right)$. This shows that all slices $G^{\prime}(k)$ with $k$ divisible by $z^{\prime}$ are actually translates of each other; hence, each of them is a coset of the subgroup $K:=G^{\prime}(0) \leq H$.

Fix arbitrarily $g \in G^{\prime}\left(z^{\prime}\right)$. For any integer $k$ divisible by $z^{\prime}$, we have $\left(k / z^{\prime}\right) g \in G^{\prime} \cap$ $(k+H)=G^{\prime}(k)$. It follows that $G^{\prime}(k)=\left(k / z^{\prime}\right) g+K$ for any integer $k$ with $z^{\prime} \mid k$. As a result, $G^{\prime}=\langle g\rangle+K$.

We need the following lemma in the spirit of [BP18].

Lemma 5. Suppose that $B$ and $C$ are finite, nonempty integer sets, and write $m:=|B|$ and $B=\left\{b_{1}, \ldots, b_{m}\right\}$, where the elements of $B$ are numbered in an arbitrary order. Then 
there exist $c_{2}, \ldots, c_{m} \in C$ such that the sums $b_{2}+c_{2}, \ldots, b_{m}+c_{m}$ are distinct from each other and from the elements of the set $b_{1}+C$.

Proof. The proof follows the line of reasoning of [BP18].

Let $n:=|C|$ and consider the family of $m+n-1$ sets

$$
\begin{aligned}
& b_{1}+C, \ldots, b_{1}+C \quad(n \text { sets }) \\
& b_{2}+C, \ldots, b_{m}+C \quad(m-1 \text { sets })
\end{aligned}
$$

Following Balasubramanian and Pandey, we use the Hall marriage theorem to show that this set family has a system of distinct representatives; clearly, this will imply the result.

Suppose thus that for some $1 \leq k \leq m+n-1$ we are given a subsystem $\mathcal{S}$ of $k$ sets from among those listed above, and show, to verify the hypothesis of Hall's theorem, that $\left|\cup_{S \in \mathcal{S}} S\right| \geq k$. Let $B^{\prime} \subseteq B$ consist of all those elements $b_{i} \in B(1 \leq i \leq m)$ such that at least one of the sets in $\mathcal{S}$ has the form $b_{i}+C$. Then $\cup_{S \in \mathcal{S}}=B^{\prime}+C$ and we thus want to show that $\left|B^{\prime}+C\right| \geq k$. Since $\left|B^{\prime}+C\right| \geq\left|B^{\prime}\right|+|C|-1$, it suffices to show that $\left|B^{\prime}\right|+n-1 \geq k$. Indeed, this inequality is trivial for $k \leq n$, while for $k \geq n$ it becomes evident upon writing $k=n+\varkappa, \varkappa \geq 0$ and observing any $n+\varkappa$ sets under consideration determine at least $\varkappa+1=k-n+1$ elements $b_{i}$.

Corollary 3. Suppose that the abelian group $G$ has the direct sum decomposition $G=$ $\mathbb{Z} \oplus H$ with $H<G$ finite. Let $B, C$ be finite, nonempty subsets of $G$. If $m$ and $n$ denote the sizes of the images of $B$ and $C$, respectively, under the projection $G \rightarrow \mathbb{Z}$ along $H$, then

$$
|B+C| \geq\left(1+\frac{n-1}{m}\right)|B| .
$$

Proof. Denote by $\psi$ the projection in question, and write $\psi(B):=\left\{b_{1}, \ldots, b_{m}\right\}$, where $b_{1}$ is chosen so that $\left|\psi^{-1}\left(b_{1}\right) \cap B\right| \geq|B| / m$; otherwise, the elements of $\psi(B)$ are numbered arbitrarily. Let $B_{i}:=\psi^{-1}\left(b_{i}\right) \cap B(1 \leq i \leq m)$. By Lemma 5 as applied to the sets $\psi(B)$ and $\psi(C)$, there are (not necessarily distinct) elements $c_{2}, \ldots, c_{m} \in \psi(C)$ such that all sums $b_{2}+c_{2}, \ldots, b_{m}+c_{m}$ are distinct from each other and from the elements of the set $b_{1}+\psi(C)$. Consequently, the sumsets $B_{2}+\left(\psi^{-1}\left(c_{2}\right) \cap C\right), \ldots, B_{m}+\left(\psi^{-1}\left(c_{m}\right) \cap C\right)$ are pairwise disjoint, and they are also disjoint from each of the $n$ sumsets $B_{1}+\left(\psi^{-1}(c) \cap\right.$ $C), c \in \psi(C)$. As a result,

$$
\begin{aligned}
|B+C| \geq \sum_{i=2}^{m} \mid B_{i} & +\left(\psi^{-1}\left(c_{i}\right) \cap C\right)\left|+\sum_{c \in \psi(C)}\right| B_{1}+\left(\psi^{-1}(c) \cap C\right) \mid \\
& \geq\left|B_{2}\right|+\cdots+\left|B_{m}\right|+n\left|B_{1}\right|=|B|+(n-1)\left|B_{1}\right| \geq|B|+\frac{n-1}{m}|B| .
\end{aligned}
$$




\section{Basic Estimates}

We collect in this section several basic estimates used in the proof of Theorem 2 .

Suppose that $A$ is a finite subset of the group $G=\mathbb{Z} \oplus H$, where $H<G$ is finite abelian. For each $z \in \mathbb{Z}$, let $A_{z}:=A \cap(z+H)$, and write $B:=\left\{z \in \mathbb{Z}: A_{z} \neq \varnothing\right\}$; that is, $B$ is the image of $A$ under the projection of $G$ onto $\mathbb{Z}$ along $H$. Suppose, furthermore, that $\min B=0, \max B=l>0,0 \in A_{0}$, and $\delta \in A_{l}$. Finally, write $n:=|B|, \sigma:=\left|A_{0}\right|+\left|A_{l}\right|$, and $A^{*}:=A_{0} \cap\left(A_{l}-\delta\right)$.

Lemma 6. We have $|2 A|+\left|A^{*}\right| \geq \sigma n$.

Proof. Considering the projections of the "slices" $A_{b}$ onto $\mathbb{Z}$, we get

$$
\begin{aligned}
|2 A| & \geq \sum_{\substack{z \in B \\
z<l}}\left|A_{0}+A_{z}\right|+\left|A_{0}+A_{l}\right|+\sum_{\substack{z \in B \\
z>0}}\left|A_{z}+A_{l}\right| \\
& \geq(n-1)\left|A_{0}\right|+\left|A_{0}+A_{l}\right|+(n-1)\left|A_{l}\right| .
\end{aligned}
$$

To estimate the sum $A_{0}+A_{l}$ we notice that both $A_{0}+\delta$ and $A_{l}$ are subsets of $A_{0}+A_{l}$, whence

$$
\left|A_{0}+A_{l}\right| \geq\left|\left(A_{0}+\delta\right) \cup A_{l}\right|=\left(\left|A_{0}\right|+\left|A_{l}\right|\right)-\left|A^{*}\right| .
$$

Combining these estimates yields the sought inequality.

Corollary 4. Let $\tau:=|2 A| /|A|$. If $\tau<3\left(1-\frac{1}{n}\right)$, then

$$
\begin{gathered}
(3-\tau)\left(\tau|A|+\left|A^{*}\right|\right)>3 \sigma, \\
3|A|-|2 A|>\sigma
\end{gathered}
$$

and

$$
|2 A|<3|A|-2\left|A^{*}\right|
$$

Proof. To prove (8), we multiply the inequality of the lemma by the inequality $3-\tau>\frac{3}{n}$ following from $\tau<3\left(1-\frac{1}{n}\right)$, and then substitute $|2 A|=\tau|A|$.

For (9), we use (8) to get

$$
\begin{aligned}
3|A|-|2 A|=(3-\tau)|A|>\frac{1}{\tau}\left(3 \sigma-(3-\tau)\left|A^{*}\right|\right) & =\frac{3}{\tau} \sigma-\left(\frac{3}{\tau}-1\right)\left|A^{*}\right| \\
\geq & \frac{3}{\tau} \sigma-\left(\frac{3}{\tau}-1\right) \cdot \frac{\sigma}{2}=\frac{1}{2}\left(\frac{3}{\tau}+1\right) \sigma>\sigma .
\end{aligned}
$$

Finally, (10) follows from

$$
|2 A|<3|A|-\sigma \leq 3|A|-2\left|A^{*}\right|
$$




\section{The TWO-COSET CASE}

In this section we prove a result which is easily seen to imply the special case of Theorem 2 where the set $A$ is contained in a union of two cosets of a subgroup $F<G$ (but not contained in a single coset of either $F$ or a subgroup containing $F$ as an index-2 subgroup).

Proposition 1. Suppose that the abelian group $G$ has the direct sum decomposition $G=\mathbb{Z} \oplus H$ with $H<G$ finite. Let $A_{1}, A_{2} \subset G$ be finite, nonempty subsets of $G$, and for $i \in\{1,2\}$ let $n_{i}:=\left|\psi\left(A_{i}\right)\right|$, where $\psi: G \rightarrow \mathbb{Z}$ is the projection along $H$. Then

$$
\left|2 A_{1}\right|+\left|A_{1}+A_{2}\right|+\left|2 A_{2}\right| \geq 3\left(1-\frac{1}{n_{1}+n_{2}}\right)\left(\left|A_{1}\right|+\left|A_{2}\right|\right) .
$$

Example 2. If, for $i \in\{1,2\}$, we let $A_{i}=P_{i}+K$, where $P_{i}$ are arithmetic progressions with the same difference not contained in $H$, and where $K \leq H$, then $n_{i}=\left|P_{i}\right|$ and

$$
\left|2 A_{1}\right|+\left|A_{1}+A_{2}\right|+\left|2 A_{2}\right|=3\left(\left|P_{1}\right|+\left|P_{2}\right|-1\right)|K|=3\left(1-\frac{1}{n_{1}+n_{2}}\right)\left(\left|A_{1}\right|+\left|A_{2}\right|\right) .
$$

This shows that the estimate of the proposition is best possible.

Proof of Proposition 1. Recall that for a subset $S$ of an abelian group, by $\pi(S)$ we denote the period of $S$; see Section 3 .

For $i \in\{1,2\}$, we have $\pi\left(2 A_{i}\right) \leq H$ (as $2 A_{i}$ are finite), and $\left|\psi\left(2 A_{i}\right)\right| \geq 2 n_{i}-1$, whence

$$
\left|2 A_{i}\right| \geq\left(2 n_{i}-1\right)\left|\pi\left(2 A_{i}\right)\right| \text {. }
$$

On the other hand, by Kneser's theorem (Section 3)

$$
\left|2 A_{i}\right| \geq 2\left|A_{i}\right|-\left|\pi\left(2 A_{i}\right)\right|
$$

Multiplying the latter inequality by $2 n_{i}-1$ and adding the former to the result (to cancel out the term $\left.\left|\pi\left(2 A_{i}\right)\right|\right)$ we get

$$
\left|2 A_{i}\right| \geq\left(2-\frac{1}{n_{i}}\right)\left|A_{i}\right|
$$

Similarly, letting $n:=n_{1}+n_{2}$ and observing that

$$
\left|\psi\left(A_{1}+A_{2}\right)\right|=\left|\psi\left(A_{1}\right)+\psi\left(A_{2}\right)\right| \geq n_{1}+n_{2}-1=n-1,
$$

we get $\left|A_{1}+A_{2}\right| \geq(n-1)\left|\pi\left(A_{1}+A_{2}\right)\right|$ and $\left|A_{1}+A_{2}\right| \geq\left|A_{1}\right|+\left|A_{2}\right|-\left|\pi\left(A_{1}+A_{2}\right)\right|$, implying

$$
\left|A_{1}+A_{2}\right| \geq\left(1-\frac{1}{n}\right)\left(\left|A_{1}\right|+\left|A_{2}\right|\right)
$$

In view of (11), it suffices to show that

$$
\left|A_{1}+A_{2}\right| \geq\left(1+\frac{1}{n_{1}}-\frac{3}{n}\right)\left|A_{1}\right|+\left(1+\frac{1}{n_{2}}-\frac{3}{n}\right)\left|A_{2}\right| .
$$

Assuming for definiteness that $n_{1} \leq n_{2}$, we distinguish two cases. 
If $\left|A_{1}\right| / n_{1} \leq\left|A_{2}\right| / n_{2}$, then we apply (12), reducing the inequality to prove to

$$
\left(1-\frac{1}{n}\right)\left(\left|A_{1}\right|+\left|A_{2}\right|\right) \geq\left(1+\frac{1}{n_{1}}-\frac{3}{n}\right)\left|A_{1}\right|+\left(1+\frac{1}{n_{2}}-\frac{3}{n}\right)\left|A_{2}\right| .
$$

This can be rewritten as

$$
\frac{1}{n_{1}}\left|A_{1}\right|+\frac{1}{n_{2}}\left|A_{2}\right| \leq 2 \frac{\left|A_{1}\right|+\left|A_{2}\right|}{n_{1}+n_{2}}
$$

and, furthermore, as

$$
\left(n_{1}-n_{2}\right)\left(\frac{\left|A_{1}\right|}{n_{1}}-\frac{\left|A_{2}\right|}{n_{2}}\right) \geq 0,
$$

which is true by our present assumptions $n_{1} \leq n_{2}$ and $\left|A_{1}\right| / n_{1} \leq\left|A_{2}\right| / n_{2}$.

Assume now that, in addition to $n_{1} \leq n_{2}$, we have

$$
\left|A_{1}\right| / n_{1}>\left|A_{2}\right| / n_{2} \text {. }
$$

By Corollary 3 (applied with $B=A_{1}$ and $C=A_{2}$ ),

$$
\left|A_{1}+A_{2}\right| \geq \frac{n-1}{n_{1}}\left|A_{1}\right| .
$$

Substituting this estimate into (13), we see that it suffices to prove that

$$
\frac{n-1}{n_{1}}\left|A_{1}\right| \geq\left(1+\frac{1}{n_{1}}-\frac{3}{n}\right)\left|A_{1}\right|+\left(1+\frac{1}{n_{2}}-\frac{3}{n}\right)\left|A_{2}\right|
$$

that is,

$$
\left(\frac{n-2}{n_{1}}-1+\frac{3}{n}\right)\left|A_{1}\right| \geq\left(1+\frac{1}{n_{2}}-\frac{3}{n}\right)\left|A_{2}\right| .
$$

In view of (14), this will follow from

$$
\left(\frac{n-2}{n_{1}}-1+\frac{3}{n}\right) n_{1} \geq\left(1+\frac{1}{n_{2}}-\frac{3}{n}\right) n_{2}
$$

which is easily verified to hold (as an equality, in fact).

\section{Proof of Theorem 2}

Recall that we have a finite subset $A$ of the abelian group $G=\mathbb{Z} \oplus H$, where $H \leq G$ is finite. We assume that $|2 A|<3\left(1-\frac{1}{n}\right)|A|$, where $n$ is the number of elements in the image of $A$ under the projection $G \rightarrow \mathbb{Z}$ along $H$, and we want to show that there exist an arithmetic progression $P \subseteq G$ and a subgroup $K \leq H$ such that $A \subseteq P+K$ and $(|P|-1)|K| \leq|2 A|-|A|$. As shown in Section 1 , the estimates $3 \leq|P| \leq(\tau-1) n+1$ and the equality $|P+K|=|P||K|$ follow automatically and we disregard them for the rest of the proof. Here $\tau$ is defined by $|2 A|=\tau|A|$, so that $\tau<3\left(1-\frac{1}{n}\right)$.

Let $\psi: G \rightarrow \mathbb{Z}$ be the projection mentioned in the previous paragraph. Without loss of generality we assume that $0 \in A$ and $\min \psi(A)=0$, and we let $l:=\max \psi(A)$; thus, $A \cap(z+H)=\varnothing$ for $z<0$ and also for $z>l$, while the sets $A_{0}:=A \cap H$ and $A_{l}:=A \cap(l+H)$ are nonempty. 
Fix arbitrarily an element $\delta \in A_{l}$, and let $A^{*}:=A_{0} \cap\left(A_{l}-\delta\right)$ and $\sigma:=\left|A_{0}\right|+\left|A_{l}\right|$. Notice that $0 \in A^{*}, \sigma \geq 2\left|A^{*}\right|$, and $\left|A_{0} \cup\left(A_{l}-\delta\right)\right|=\sigma-\left|A^{*}\right|$.

For a subgroup $L \leq G$, by $\varphi_{L}$ we denote the canonical homomorphism of $G$ onto the quotient group $G / L$. Let $\Delta:=\langle\delta\rangle \leq G$. We adopt a special notation for the homomorphism $\varphi_{\Delta}$, which is particularly important for our argument: whenever $s$ denotes an element of $G$, by $\bar{s}$ we denote the image of $s$ under $\varphi_{\Delta}$, and similarly for sets: $\bar{S}=\varphi_{\Delta}(S), S \subseteq G$. Thus, for instance, $\bar{A}=\varphi_{\Delta}(A)$ and $\overline{2 A}=\varphi_{\Delta}(2 A)=2 \bar{A}$.

To make the proof easier to follow, we split it into several parts.

6.1. Deficiency and the induction framework. We use induction on $|H|$, the base case $|H|=1$ being Freiman's $(3 n-3)$-theorem (see Section 1). Suppose that $|H| \geq 2$.

Given a subset $S \subseteq G$ and a subgroup $L \leq G$, both finite, we define the deficiency of $S$ on a coset $g+L \subseteq G$ by

$$
\mathrm{d}(S, g+L):= \begin{cases}|(g+L) \backslash S| & \text { if } S \cap(g+L) \neq \varnothing \\ 0 & \text { if } S \cap(g+L)=\varnothing\end{cases}
$$

notice that in the first case we can also write $\mathrm{d}(S, g+L)=|L|-|(g+L) \cap S|$. The total deficiency of $S$ with respect to $L$ is

$$
\mathrm{D}(S, L):=|(S+L) \backslash S|
$$

equivalently,

$$
\mathrm{D}(S, L)=\sum_{g+L} \mathrm{~d}(S, g+L),
$$

where the sum extends over all $L$-cosets having a nonempty intersection with $S$.

Suppose that $L \leq H$ is a nonzero finite subgroup with

$$
\mathrm{D}(2 A, L) \leq \mathrm{D}(A, L) \text {. }
$$

Then, letting $T:=3\left(1-\frac{1}{n}\right)$,

$$
|2(A+L)| \leq|A+L|+|2 A|-|A|<|A+L|+(T-1)|A| \leq T|A+L|
$$

that is, writing $\widetilde{G}:=G / L \cong(H / L) \oplus \mathbb{Z}, \widetilde{A}:=\varphi_{L}(A)$, and $\widetilde{2 A}:=\varphi_{L}(2 A)$, we have $|2 \widetilde{A}|<3\left(1-\frac{1}{n}\right)|\widetilde{A}|$. Applying the induction hypothesis to the subset $\widetilde{A} \subseteq \widetilde{G}$, we conclude that there are an arithmetic progression $\widetilde{P} \subseteq \widetilde{G}$ and a subgroup $\widetilde{K} \leq \widetilde{H}:=H / L$ such that $\widetilde{A} \subseteq \widetilde{P}+\widetilde{K}$ and $(|\widetilde{P}|-1)|\widetilde{K}| \leq|2 \widetilde{A}|-|\widetilde{A}|$. Let $K:=\varphi_{L}^{-1}(\widetilde{K})$; thus, $L \leq K \leq H$ and $|K|=|\bar{L}||\widetilde{K}|$. Also, it is easily seen that $\varphi_{L}^{-1}(\widetilde{P})=P+L$ where $P \subseteq G$ is an arithmetic progression with $|P| \leq|\widetilde{P}|$. From $\widetilde{A} \subseteq \widetilde{P}+\widetilde{K}$ we derive then that $A \subseteq P+K$, and from $(|\widetilde{P}|-1)|\widetilde{K}| \leq|2 \widetilde{A}|-|\widetilde{A}|$ we get

$$
\begin{aligned}
(|P|-1)|K| & \leq(|\widetilde{P}|-1)|\widetilde{K}||L| \leq(|2 \widetilde{A}|-|\widetilde{A}|)|L| \\
& =|2 A+L|-|A+L|=|2 A|+\mathrm{D}(2 A, L)-|A|-\mathrm{D}(A, L) \leq|2 A|-|A|,
\end{aligned}
$$

completing the induction step. 
Of particular interest is the situation where $L$ is a nonzero, finite subgroup satisfying

$$
\mathrm{D}(A, L) \leq|L|-1
$$

Let in this case $m$ denote the number of $L$-cosets on which $A$ has positive deficiency, and fix $a_{1}, \ldots, a_{m} \in A$ such that $a_{1}+L, \ldots, a_{m}+L$ list all these cosets. It follows easily from (16) that there is at most one pair of indices $1 \leq i \leq j \leq m$ such that $\mathrm{d}\left(A, a_{i}+L\right)+\mathrm{d}\left(A, a_{j}+L\right) \geq|L|$, and if such a pair exists, then in fact $i=j$. By the pigeonhole principle, we have then $\mathrm{d}(2 A, g+L)=0$ for every coset $g+L$, with the possible exception of one single $L$-coset which is then of the form $2 a+L$, with some $a \in A$. This yields

$$
\mathrm{D}(2 A, L)=\mathrm{d}(2 A, 2 a+L) \leq \mathrm{d}(A, a+L) \leq \mathrm{D}(A, L)
$$

Clearly, the resulting estimate

$$
\mathrm{D}(2 A, L) \leq \mathrm{D}(A, L)
$$

remains valid also if there are no exceptional $L$-cosets.

Thus, once we are able to find a nonzero finite subgroup $L<H$ satisfying either (15) or (16), we can complete the proof applying the induction hypothesis.

As a result, we can assume that for any nonempty subsets $A^{\prime}, A^{\prime \prime} \subseteq A$ with $A=A^{\prime} \cup A^{\prime \prime}$,

$$
\left|A^{\prime}+A^{\prime \prime}\right| \geq\left|A^{\prime}\right|+\left|A^{\prime \prime}\right|-1
$$

for if this fails to hold, then letting $L:=\pi\left(A^{\prime}+A^{\prime \prime}\right)$, by Kneser's theorem we have $|L| \geq 2$ and $\left|A^{\prime}+L\right|+\left|A^{\prime \prime}+L\right|-|L|=\left|A^{\prime}+A^{\prime \prime}\right| \leq\left|A^{\prime}\right|+\left|A^{\prime \prime}\right|-2$, whence

$$
\mathrm{D}(A, L) \leq \mathrm{D}\left(A^{\prime}, L\right)+\mathrm{D}\left(A^{\prime \prime}, L\right) \leq|L|-2
$$

(for the first inequality, notice that $\mathrm{d}(A, g+L) \leq \mathrm{d}\left(A^{\prime}, g+L\right)+\mathrm{d}\left(A^{\prime \prime}, g+L\right)$ for any coset $g+L$, which follows from the assumption $\left.A^{\prime}, A^{\prime \prime} \subseteq A=A^{\prime} \cup A^{\prime \prime}\right)$.

In particular, we assume that $|A+S| \geq|A|+|S|-1$ for any nonempty subset $S \subseteq A$. As an important special case,

$$
\left|A+A^{*}\right| \geq|A|+\left|A^{*}\right|-1 \text {. }
$$

6.2. The set $\bar{A}$ has small doubling. The quantity $\left|A^{*}\right|$ can be interpreted as the number of representations of $\delta$ as a difference of two elements of $A$. Generally, for a set $S \subseteq G$ and an element $g \in G$, denote by $r_{S}(g)$ the number of representations of $g$ as a difference of two elements of $S$; thus, for instance, $\left|A^{*}\right|=r_{A}(\delta)$. Clearly, every $\Delta$-coset intersects $A$ by at most two elements, and if the intersection contains exactly two elements, then the two elements differ by $\delta$. It follows that

$$
|A|=|\bar{A}|+r_{A}(\delta)=|\bar{A}|+\left|A^{*}\right| .
$$

Similarly, since $\bar{s}_{1}=\bar{s}_{2}$ for any $s_{1}, s_{2} \in 2 A$ with $s_{2}-s_{1}=\delta$, we have $|2 A| \geq|2 \bar{A}|+r_{2 A}(\delta)$. Furthermore, $r_{2 A}(\delta) \geq\left|A+A^{*}\right|$ as to any $a \in A$ and $a^{*} \in A^{*}$ there corresponds the 
representation $\left(\left(a^{*}+\delta\right)+a\right)-\left(a^{*}+a\right)=\delta$, and the sum $a+a^{*}$ is uniquely determined by this representation. Therefore,

$$
|2 A| \geq|2 \bar{A}|+\left|A+A^{*}\right|
$$

We now claim that

$$
|2 \bar{A}|<2|\bar{A}|-1
$$

In view of $|2 \bar{A}| \leq|2 A|-\left|A+A^{*}\right| \leq \tau|A|-|A|-\left|A^{*}\right|+1$ and $|\bar{A}|=|A|-\left|A^{*}\right|$ (cf. (19), (17), and (18)), it suffices to show that

$$
\tau|A|-|A|-\left|A^{*}\right|+1<2|A|-2\left|A^{*}\right|-1 ;
$$

that is,

$$
(3-\tau)|A|>\left|A^{*}\right|+2 .
$$

To this end we notice that, by (8) and in view of $\left|A^{*}\right| \leq \min \left\{\left|A_{0}\right|,\left|A_{l}\right|\right\}$,

$$
(3-\tau)\left(\tau|A|+\left|A^{*}\right|\right)>3\left(\left|A_{0}\right|+\left|A_{l}\right|\right) \geq 6\left|A^{*}\right| .
$$

Consequently,

$$
(3-\tau)|A|>\left(\frac{3}{\tau}+1\right)\left|A^{*}\right|>2\left|A^{*}\right|
$$

which proves $(21)$ in the case where $\left|A^{*}\right| \geq 2$. In the remaining case $\left|A^{*}\right|=1$, we obtain (21) as an immediate corollary of $|A| \geq n$ and $\tau<3\left(1-\frac{1}{n}\right)$.

Thus, (20) is established, and from Kneser's theorem it follows that the period $\bar{F}:=$ $\pi(2 \bar{A})$ is a nonzero subgroup of the quotient group $G / \Delta$, and also, in view of $2|\bar{A}+\bar{F}|-$ $|\bar{F}|=|2 \bar{A}| \leq 2|\bar{A}|-2$, that

$$
\mathrm{D}(\bar{A}, \bar{F}) \leq \frac{1}{2}|\bar{F}|-1 .
$$

We let $F:=\varphi_{\Delta}^{-1}(\bar{F})$, so that $\bar{F}=\varphi_{\Delta}(F)$ and $\Delta \leq F \leq G$.

Observing that $0 \in A$ implies $\bar{A}+\bar{F} \subseteq 2 \bar{A}+\bar{F}=2 \bar{A}$, we denote by $N$ the number of $\bar{F}$-cosets contained in $2 \bar{A}$, but not in $\bar{A}+\bar{F}$; that is,

$$
N=(|2 \bar{A}|-|\bar{A}+\bar{F}|) /|\bar{F}| \text {. }
$$

Combining $|2 \bar{A}|-|\bar{A}+\bar{F}|=N|\bar{F}|$ and $|2 \bar{A}|=2|\bar{A}+\bar{F}|-|\bar{F}|$, we get

$$
|\bar{A}+\bar{F}|=(N+1)|\bar{F}| \text { and }|2 \bar{A}|=(2 N+1)|\bar{F}| .
$$

Let $K:=F \cap H$.

6.3. The case where $N=0$. If $N=0$, then $\bar{A}+\bar{F}=2 \bar{A}$. Adding $\bar{A}$ to both sides we get $2 \bar{A}=2 \bar{A}+\bar{A}$, showing that $\bar{A} \subseteq \pi(2 \bar{A})=\bar{F}$. Combining this with $\bar{A}+\bar{F}=2 \bar{A}$, we conclude that $2 \bar{A}=\bar{F}$. Thus, $2 A+\Delta=F$ and, by Lemma 4 ,

$$
A \subseteq 2 A+\Delta=F=\langle g\rangle+K
$$

with some $g \in G$. Notice that $g \notin H$, as otherwise we would have $A \subseteq H$, and hence $n=1$. 
Let $P:=\langle g\rangle \cap \psi^{-1}([0, l])$, so that $A \subseteq P+K$. Since $\psi^{-1}([0, l))$ contains exactly one representative out of every $\Delta$-coset, we also have

$$
\begin{aligned}
|2 \bar{A}| & =\left|\varphi_{\Delta}(2 A)\right| \\
& =\left|\varphi_{\Delta}(2 A+\Delta)\right| \\
& =\left|(2 A+\Delta) \cap \psi^{-1}([0, l))\right| \\
& =\left|(\langle g\rangle+K) \cap \psi^{-1}([0, l))\right| \\
& =\left|\langle g\rangle \cap \psi^{-1}([0, l))\right||K| \\
& =\left|\langle g\rangle \cap \psi^{-1}([0, l])\right||K|-\left|\langle g\rangle \cap \psi^{-1}(l)\right||K| \\
& =(|P|-1)|K|,
\end{aligned}
$$

the middle equality following from (24), and the last equality from

$$
\varnothing \neq A \cap \psi^{-1}(l) \subseteq(\langle g\rangle+K) \cap \psi^{-1}(l)=\left(\langle g\rangle \cap \psi^{-1}(l)\right)+K
$$

and the resulting $\langle g\rangle \cap \psi^{-1}(l) \neq \varnothing$. Consequently, (19) yields $(|P|-1)|K| \leq|2 A|-|A|$, completing the proof in the present case.

We thus assume for the remaining part of the argument $\mathrm{t}=$ hat $N>0$; that is

$$
\bar{A}+\bar{F} \subsetneq 2 \bar{A} .
$$

Therefore, $2 \bar{A}$ is not a subgroup (if it were, we would have $\bar{F}=\pi(2 \bar{A})=2 \bar{A}$ implying $\bar{A}+\bar{F} \supseteq 2 \bar{A})$.

6.4. The case where $N=1$. If $N=1$, then $\bar{A}+\bar{F}$ is a union of exactly two $\bar{F}$-cosets, and $2 \bar{A}$ is a union of exactly three $\bar{F}$-cosets. Since $0 \in A$, we derive that $A=A_{1} \cup\left(g+A_{2}\right)$, where $A_{1}, A_{2} \subseteq F$ are nonempty and finite, and where $g \in G$ satisfies $2 g \notin F$, as a result of $2 \bar{A}$ being a union of three $\bar{F}$-cosets. Write $n_{i}:=\left|\psi^{-1}\left(A_{i}\right)\right|, i \in\{1,2\}$, so that $n:=\left|\psi^{-1}(A)\right| \leq n_{1}+n_{2}$. By Proposition 1, we have then

$$
\begin{aligned}
|2 A| & =\left|2 A_{1}\right|+\left|A_{1}+A_{2}\right|+\left|2 A_{2}\right| \\
& \geq 3\left(1-\frac{1}{n_{1}+n_{2}}\right)\left(\left|A_{1}\right|+\left|A_{2}\right|\right) \\
& \geq 3\left(1-\frac{1}{n}\right)|A|,
\end{aligned}
$$

a contradiction.

Let $\bar{H}:=\varphi_{\Delta}(H)$ and, following our standard convention, write $\bar{K}:=\varphi_{\Delta}(K)$. We split the remaining case $N \geq 2$ into two further subcases: that where $\bar{K}$ is a proper subgroup of $\bar{F}$ (which, by Corollary 2, is equivalent to any of $\bar{F} \not \leq \bar{H}$ and $F \not \leq H+\Delta$ ), and that where $\bar{K}=\bar{F}$ (equivalently, $\bar{F} \leq \bar{H}, F \leq H+\Delta$, or $F=K \oplus \Delta$ ). 
6.5. The case where $N \geq 2$ and $\bar{K} \supsetneqq \bar{F}$. We show that in this case

$$
|2 A \backslash A| \geq 2|\bar{A}|
$$

in view of (18) and (10), this will give

$$
|2 A|-|A| \geq 2|\bar{A}|=2|A|-2\left|A^{*}\right|>2|A|-(3-\tau)|A|=(\tau-1)|A|,
$$

a contradiction.

To prove (25), we partition the elements $s \in 2 A \backslash A$ into two groups, according to whether $\bar{s}=\varphi_{\Delta}(s)$ lies in $\bar{A}+\bar{F}$.

For the first group we have the estimate

$$
|\{s \in 2 A \backslash A: \bar{s} \in \bar{A}+\bar{F}\}| \geq|\bar{A}+\bar{F}|
$$

for, $\bar{A}+\bar{F} \subseteq 2 \bar{A}$ shows that for every element $\bar{s} \in \bar{A}+\bar{F}$, the set $\left\{s \in 2 A: \varphi_{\Delta}(s)=\bar{s}\right\}$ is nonempty, and the (unique) element of this set with the largest value of $\psi(s)$ does not lie in $A$ as $s \in A$ implies $s+\delta \in 2 A$, because of $\delta \in A$. (This argument shows that, indeed, for any subset $\bar{S} \subseteq 2 \bar{A}$ there are at least $|\bar{S}|$ elements $s \in 2 A \backslash A$ such that $\bar{s} \in \bar{S}$.)

To complete the treatment of the case $\bar{K} \supsetneqq \bar{F}$, we show that

$$
T:=|\{s \in 2 A \backslash A: \bar{s} \notin \bar{A}+\bar{F}\}| \geq 2|\bar{A}|-|\bar{A}+\bar{F}| .
$$

(Notice that the trivial estimate would be $T \geq|2 \bar{A}|-|\bar{A}+\bar{F}|$.)

The set $(2 \bar{A}) \backslash(\bar{A}+\bar{F})$ is a union of $\bar{F}$-cosets, and we find $a_{1}, \ldots, a_{N}, b_{1}, \ldots, b_{N} \in A$ such that the cosets in question are $\bar{a}_{i}+\bar{b}_{i}+\bar{F}, i \in[1, N]$.

Let

$$
A_{i}:=A \cap\left(a_{i}+F\right) \text { and } B_{i}:=A \cap\left(b_{i}+F\right), \quad i \in[1, N] .
$$

By Corollary 2 we have $\bar{A}_{i}=\bar{A} \cap\left(\bar{a}_{i}+\bar{F}\right)$ and $\bar{B}_{i}=\bar{A} \cap\left(\bar{b}_{i}+\bar{F}\right)$, and it follows that

$$
\left|A_{i}\right| \geq\left|\bar{A}_{i}\right|=\left|\bar{a}_{i}+\bar{F}\right|-\left|\left(\bar{a}_{i}+\bar{F}\right) \backslash \bar{A}\right|=|\bar{F}|-\mathrm{d}\left(\bar{A}, \bar{a}_{i}+\bar{F}\right), \quad i \in[1, N]
$$

and, similarly,

$$
\left|B_{i}\right| \geq\left|\bar{B}_{i}\right|=\left|\bar{b}_{i}+\bar{F}\right|-\left|\left(\bar{b}_{i}+\bar{F}\right) \backslash \bar{A}\right|=|\bar{F}|-\mathrm{d}\left(\bar{A}, \bar{b}_{i}+\bar{F}\right), \quad i \in[1, N] .
$$

Since $\varphi_{\Delta}\left(A_{i}+B_{i}\right)=\bar{A}_{i}+\bar{B}_{i} \subseteq \bar{a}_{i}+\bar{b}_{i}+\bar{F} \subseteq(2 \bar{A}) \backslash(\bar{A}+\bar{F})$ by the choice of $a_{i}$ and $b_{i}$, we have

$$
T=\sum_{i=1}^{N}\left|\left\{s \in 2 A \backslash A: \bar{s} \in \bar{a}_{i}+\bar{b}_{i}+\bar{F}\right\}\right| \geq \sum_{i=1}^{N}\left|A_{i}+B_{i}\right| .
$$

By Lemma 2 as applied to the subset $\widetilde{A}:=(\bar{A}+\bar{F}) / \bar{F}$ of the quotient group $\bar{G} / \bar{F}$, we can assume that each $\bar{F}$-coset from $\bar{A}+\bar{F}$ appears among the $2 N \operatorname{cosets} \bar{a}_{1}+\bar{F}, \ldots, \bar{b}_{N}+\bar{F}$ at most $N$ times, except if there is a subgroup $\widetilde{L} \leq \bar{G} / \bar{F}$ and an element $\widetilde{c} \in \bar{G} / \bar{F}$ with $2 \widetilde{c} \notin \widetilde{L}$ such that either $\widetilde{A}=\widetilde{L} \cup\{\widetilde{c}\}$, or $\widetilde{A}=(\widetilde{c}+\widetilde{L}) \cup\{0\}$. In this exceptional situation $A$ meets exactly two cosets of the subgroup $L=\varphi_{F}^{-1}(\widetilde{L})$, while $2 A$ meets exactly three cosets of this subgroup. As a result, we can apply Proposition 1, exactly as in the case $N=1$ considered above, to get $|2 A| \geq 3\left(1-\frac{1}{n}\right)|A|$. 
Addressing now case i) of Lemma 2, assume that each $\bar{F}$-coset from $\bar{A}+\bar{F}$ appears among $\bar{a}_{1}+\bar{F}, \ldots, \bar{b}_{N}+\bar{F}$ at most $N$ times.

Since $A_{i}+B_{i}$ is contained in an $F$-coset, we have $\pi\left(A_{i}+B_{i}\right) \leq F$, and since $A_{i}+B_{i}$ is finite, $\pi\left(A_{i}+B_{i}\right) \leq H$; as a result, $\pi\left(A_{i}+B_{i}\right) \leq F \cap H=K$. Consequently, by (28), Kneser's theorem, (26), and (27),

$$
T \geq 2 N|\bar{F}|-\sum_{i=1}^{N}\left(\mathrm{~d}\left(\bar{A}, \bar{a}_{i}+\bar{F}\right)+\mathrm{d}\left(\bar{A}, \bar{b}_{i}+\bar{F}\right)\right)-|K| N .
$$

Recalling that each $\bar{F}$-coset from $\bar{A}+\bar{F}$ appears at most $N$ times among $\bar{a}_{1}+\bar{F}, \ldots, \bar{b}_{N}+$ $\bar{F}$, we get

$$
T \geq 2 N|\bar{F}|-N \mathrm{D}(\bar{A}, \bar{F})-|K| N \geq\left(\frac{3}{2}|\bar{F}|-\mathrm{D}(\bar{A}, \bar{F})\right) N
$$

(as $\bar{K} \lessgtr \bar{F}$ yields $|K|=|\bar{K}| \leq \frac{1}{2}|\bar{F}|$ ). Therefore, by (22), (23), and the definition of the total deficiency,

$$
\begin{aligned}
T & \geq(N+1)|\bar{F}|-2 \mathrm{D}(\bar{A}, \bar{F})+(N-2)\left(\frac{1}{2}|\bar{F}|-\mathrm{D}(\bar{A}, \bar{F})\right) \\
& \geq(N+1)|\bar{F}|-2 \mathrm{D}(\bar{A}, \bar{F}) \\
& =|\bar{A}|-\mathrm{D}(\bar{A}, \bar{F}) \\
& =2|\bar{A}|-|\bar{A}+\bar{F}| .
\end{aligned}
$$

As explained above, this leads to a contradiction.

6.6. The case where $N \geq 2$ and $\bar{K}=\bar{F}$. As shown above, in this case $\bar{F} \leq \bar{H}$, $F \leq H+\Delta$, and $F=K \oplus \Delta$; notice that this implies $|\bar{F}|=|\bar{K}|=|K|$.

Let $A^{\circ}:=A \backslash\left(A_{0} \cup A_{l}\right)$; loosely speaking, $A^{\circ}$ is the "middle part" of $A$.

Claim 1. We have $A_{0} \subseteq K$ and $A_{l} \subseteq \delta+K$; that is, each of the sets $A_{0}$ and $A_{l}$ is contained in a single $K$-coset.

Proof. From (19), Kneser's theorem, (17), and (18), we have

$$
\begin{aligned}
|2 A| \geq(2|\bar{A}+\bar{F}|-|\bar{F}|)+(|A| & \left.+\left|A^{*}\right|-1\right) \\
& \geq 2|\bar{A}|-|\bar{F}|+|A|+\left|A^{*}\right|-1=3|A|-\left|A^{*}\right|-|\bar{F}|-1 .
\end{aligned}
$$

Combining this estimate with (9), we get

$$
\sigma \leq\left|A^{*}\right|+|\bar{F}| .
$$

On the other hand,

$$
|H \cap(A+\Delta)| \geq\left|\varphi_{\Delta}(H \cap(A+\Delta))\right|=\left|\varphi_{\Delta}(H) \cap \varphi_{\Delta}(A+\Delta)\right|=|\bar{H} \cap \bar{A}|
$$

by Lemma 3. Observing that the left-hand side is

$$
|(H \cap A) \cup(H \cap(A-\delta))|=\left|A_{0} \cup\left(A_{l}-\delta\right)\right|=\sigma-\left|A^{*}\right|,
$$


and using (29), we obtain

$$
|\bar{H} \cap \bar{A}| \leq \sigma-\left|A^{*}\right| \leq|\bar{F}| .
$$

Assuming now for a contradiction that, say, $A_{0}$ intersects more than one $K$-coset, fix $a_{1}, a_{2} \in A_{0}$ which are distinct modulo $K$. Since $\bar{a}_{1}, \bar{a}_{2} \in \bar{H}$ are then distinct modulo $\bar{K}=\bar{F}$, in view of $(22)$ and the assumption $\bar{F} \leq \bar{H}$ we get

$$
\begin{aligned}
\frac{1}{2}|\bar{F}| & >\mathrm{D}(\bar{A}, \bar{F}) \\
& \geq \mathrm{d}\left(\bar{A}, \bar{a}_{1}+\bar{F}\right)+\mathrm{d}\left(\bar{A}, \bar{a}_{2}+\bar{F}\right) \\
& =2|\bar{F}|-\left(\left|\left(\bar{a}_{1}+\bar{F}\right) \cap \bar{A}\right|+\left|\left(\bar{a}_{2}+\bar{F}\right) \cap \bar{A}\right|\right) \\
& \geq 2|\bar{F}|-|(\bar{H}+\bar{F}) \cap \bar{A}| \\
& =2|\bar{F}|-|\bar{H} \cap \bar{A}| \\
& \geq|\bar{F}|,
\end{aligned}
$$

the contradiction sought.

Claim 2. We have $2 A^{\circ}+K=2 A^{\circ}$. Moreover, if $\left|A_{0}\right| \geq\left|A_{l}\right|$, then $A^{\circ}+K \subseteq 2 A$, and if $\left|A_{l}\right| \geq\left|A_{0}\right|$, then $A^{\circ}+\delta+K \subseteq 2 A$.

Proof. To prove the first assertion, we fix $a_{1}, a_{2} \in A^{\circ}$ and show that $a_{1}+a_{2}+K \subseteq 2 A^{\circ}$. For $i \in\{1,2\}$, let $A_{i}:=\left(a_{i}+F\right) \cap A$; notice that $A_{i} \subseteq a_{i}+F=a_{i}+K+\Delta$ whence, indeed, $A_{i} \subseteq a_{i}+K$. Write $S:=A_{1}+A_{2} \subseteq a_{1}+a_{2}+K$ so that $\bar{S}=\bar{A}_{1}+\bar{A}_{2}=\bar{a}_{1}+\bar{a}_{2}+\bar{F}$ in view of (22). As a result, $|S| \geq|\bar{S}|=\left|\bar{a}_{1}+\bar{a}_{2}+\bar{F}\right|=|\bar{F}|=|\bar{K}|=|K|$, leading to $S=a_{1}+a_{2}+K$; thus, $a_{1}+a_{2}+K=A_{1}+A_{2} \subseteq 2 A^{\circ}$.

Addressing the second assertion, we fix $a^{\circ} \in A^{\circ}$ and show that then either $a^{\circ}+K \subseteq 2 A$, or $a^{\circ}+\delta+K \subseteq 2 A$, according to the relation between $\left|A_{0}\right|$ and $\left|A_{l}\right|$. Write $B_{0}:=A \cap F$ and $B^{\circ}:=A \cap\left(a^{\circ}+F\right)$; equivalently, $B_{0}=A_{0} \cup A_{l}$ by Claim 1 , and $B^{\circ}=A \cap\left(a^{\circ}+K\right)$. Letting $S:=B_{0}+B^{\circ}$, in view of $B_{0} \subseteq F$ and $B^{\circ} \subseteq a^{\circ}+F$ we have then $S \subseteq 2 A \cap\left(a^{\circ}+F\right)$ and $\bar{S}=\bar{B}_{0}+\bar{B}^{\circ}$. Furthermore, from

$$
\mathrm{d}(\bar{A}, \bar{F})=|\bar{F}|-|\bar{A} \cap \bar{F}|=|\bar{F}|-\left|\bar{B}_{0}\right|
$$

and

$$
\mathrm{d}\left(\bar{A}, \bar{a}^{\circ}+\bar{F}\right)=|\bar{F}|-\left|\bar{A} \cap\left(\bar{a}^{\circ}+\bar{F}\right)\right|=|\bar{F}|-\left|\bar{B}^{\circ}\right|
$$

recalling (22) we get

$$
\left|\bar{B}_{0}\right|+\left|\bar{B}^{\circ}\right|=2|\bar{F}|-\left(\mathrm{d}(\bar{A}, \bar{F})+\mathrm{d}\left(\bar{A}, \bar{a}^{\circ}+\bar{F}\right)\right) \geq 2|\bar{F}|-\mathrm{D}(\bar{A}, \bar{F})>\frac{3}{2}|\bar{F}| .
$$

From $B_{0}=A_{0} \cup A_{l}$ we now derive

$$
\left|A_{0}\right|+\left|A_{l}\right|+\left|B^{\circ}\right| \geq\left|B_{0}\right|+\left|B^{\circ}\right| \geq\left|\bar{B}_{0}\right|+\left|\bar{B}^{\circ}\right|>\frac{3}{2}|\bar{F}| .
$$


Also, we have

$$
\left|B^{\circ}\right| \geq\left|\bar{B}^{\circ}\right|=\left|\bar{A} \cap\left(\bar{a}^{\circ}+\bar{F}\right)\right|=|\bar{F}|-\mathrm{d}\left(\bar{A}, \bar{a}^{\circ}+\bar{F}\right) \geq|\bar{F}|-\mathrm{D}(\bar{A}, \bar{F})>\frac{1}{2}|\bar{F}| .
$$

Therefore,

$$
\max \left\{\left|A_{0}\right|,\left|A_{l}\right|\right\}+\left|B^{\circ}\right| \geq \frac{1}{2}\left(\left|A_{0}\right|+\left|A_{l}\right|+\left|B^{\circ}\right|\right)+\frac{1}{2}\left|B^{\circ}\right|>\frac{3}{4}|\bar{F}|+\frac{1}{4}|\bar{F}|=|\bar{F}|=|K| .
$$

Since $B^{\circ} \subseteq a^{\circ}+K, A_{0} \subseteq K$, and $A_{l} \subseteq \delta+K$, from the pigeonhole principle we conclude that if $\left|A_{0}\right|>\left|A_{l}\right|$, then $A_{0}+B^{\circ}=a^{\circ}+K$, while if $\left|A_{l}\right| \geq\left|A_{0}\right|$, then $A_{l}+B^{\circ}=a^{\circ}+\delta+K$. The assertion follows in view of $A_{0}+B^{\circ} \subseteq 2 A$ and $A_{l}+B^{\circ} \subseteq 2 A$.

We can, eventually, complete the proof. Assuming $\left|A_{0}\right| \leq\left|A_{l}\right|$ for definiteness, by Claim 2 we have $2 A^{\circ}+K \subseteq 2 A$ and also $A^{\circ}+A_{l}+K \subseteq 2 A$; that is, the set $2 A$ has zero deficiency on all $K$-cosets with the possible exception of the cosets contained in $A_{0}+A+K$; that is, cosets of the form $a+K$ with $a \in A$. On the other hand, in view of

$$
A \cap(a+K)+A_{0} \subseteq 2 A \cap(a+K)
$$

and $|2 A \cap(a+K)| \geq|A \cap(a+K)|$ resulting from it, we have

$$
\mathrm{d}(2 A, a+K) \leq \mathrm{d}(A, a+K) .
$$

Taking the sum over the elements $a \in A$ representing the $K$-cosets contained in $A$ we get

$$
\mathrm{D}(A, K)=\sum_{a} \mathrm{~d}(A, a+K) \geq \sum_{a} \mathrm{~d}(2 A, a+K)=\mathrm{D}(2 A, K) .
$$

As noticed in Section 6.1, this completes the proof by appealing to the induction.

\section{REFERENCES}

[BP18] R. Balasubramanian and P.P. Pandey, On a Theorem of Deshouillers and Freiman, European J. Comb. 70 (2018), pp. 284-296.

[DF86] J-M. Deshouillers and G.A. Freiman, A step beyong Kneser's theorem for abelian finite groups, Proc. London Math. Soc. (3) 86 (1) (2003), 1-28.

[F62] G.A. Freiman, Inverse problems in additive number theory, VI. On the addition of finite sets, III, Izv.Vyssh. Uchebn. Zaved. Mat. 3 (1962), 151-157 (Russian).

[Kn53] M. KNeSER, Abschätzung der asymptotischen Dichte von Summenmengen, Math. Z. 58 (1953), 459-484.

[Kn55] _ Ein Satz über abelsche Gruppen mit Anwendungen auf die Geometrie der Zahlen, Math. Z. 61 (1955), 429-434.

[N96] M. Nathanson, Additive number theory. Inverse problems and the geometry of sumsets, Graduate Texts in Mathematics 165. Springer-Verlag, New York, 1996.

Email address: seva@math.haifa.ac.il

Department of mathematics, the University of Haifa at Oranim, Tivon 36006, Israel 